\title{
Neuropsychiatric Presentations and Sequelae of COVID-19: Current Evidence and Research Recommendations
}

\author{
Way Hinn Chong ${ }^{1}$, Nizrull Nasir ${ }^{2}$, Gerard Thomas Flaherty ${ }^{1 *}$ \\ ${ }^{1}$ School of Medicine, National University of Ireland Galway, Galway, Ireland \\ ${ }^{2}$ School of Medicine and Health Science, University College Dublin, Dublin, Ireland
}

Corresponding Author: Gerard Thomas Flaherty, MD, FFTM, FISTM, Professor, School of Medicine, National University of Ireland Galway, Galway, Ireland. Email: gerard.flaherty@nuigalway.ie

Received August 19, 2020; Accepted September 22, 2020; Online Published November 11, 2020

Citation: Chong WH, Nasir N, Flaherty GT. Neuropsychiatric presentations and sequelae of COVID-19: current evidence and research recommendations. Int J Travel Med Glob Health. 2021;9(1):42-43. doi:10.34172/ijtmgh.2021.08.

\section{Dear Editor,}

Global pandemics compel us to contemplate the lessons of history. The influenza pandemic of 1918-1920 gave rise to the curious neurological syndrome of encephalitis lethargica, which left its unfortunate victims with varying combinations of motionlessness, hypersomnolence, and ophthalmoplegia. There is increasing awareness during the current pandemic of coronavirus disease 2019 (COVID-19) of a range of neurologic and psychiatric manifestations which in some cases are the presenting clinical features of the infection. Furthermore, patients with chronic neurologic conditions such as multiple sclerosis are susceptible to more severe COVID-19 by virtue of their use of immunosuppressant medications. ${ }^{1}$ Some of the anti-viral drugs used in the management of COVID-19, such as remdesivir, are also known to have adverse effects which involve the nervous system. ${ }^{2}$ Increased public and physician awareness of these neuropsychiatric phenomena may aid in their earlier diagnosis and management.

A variety of neurologic symptoms have been described in patients infected with Severe acute respiratory syndrome coronavirus 2 (SARS-CoV-2). Headache, vertigo, ataxia, reduced consciousness and seizures are among the most commonly reported symptoms arising from involvement of the central nervous system. ${ }^{3}$ Guillain-Barré syndrome, an acute polyradiculopathy presenting with flaccid ascending limb weakness, has also been described. ${ }^{4}$ Agarwal et al published a case series of five patients with diverse manifestations of CNS involvement, including two 41-year-old females, one with limb weakness and aphasia resulting from an ischaemic stroke, and the other with internuclear ophthalmoplegia secondary to oedema of the corpus callosum. ${ }^{2}$

Anosmia may result from rhinitis secondary to respiratory viruses including influenza. Early in the COVID-19 pandemic, this symptom emerged as one of its more prominent presenting features, sometimes in the absence of other clinical features. A cross-sectional study from Spain found that smell and/or taste dysfunction were at least two-fold more common in COVID-19 patients than in controls. ${ }^{5}$ This chemosensory impairment mainly afflicted younger patients who did not require hospitalisation. Subclinical deficits in olfaction demonstrated by quantitative smell testing have been proposed as a sensitive biomarker for COVID-19. ${ }^{6}$ Given that olfactory receptor cells do not express the angiotensinconverting enzyme 2 (ACE2) receptor exploited by this novel coronavirus, multiple pathogenetic mechanisms for these sensory deficits have been proposed which will stimulate further investigation.

COVID-related cerebrovascular disease, in the form of ischaemic stroke, intracerebral haemorrhage and cerebral dural venous sinus thrombosis, result primarily from large vessel disease and are believed to be a reflection of the prothrombotic state caused by the endothelial damage from SARSCoV-2 infection. ${ }^{4}$ Many of the most severely ill COVID-19 patients present other risk factors for cerebrovascular disease such as diabetes, obesity and hypertension. Some of the improvement in survival rates among hospitalised COVID-19 patients may be attributed to the increased use of antiplatelet agents and anticoagulants in this patient cohort as clinical experience with this virus increases.

Postulated mechanisms of COVID-19 neurological disease include direct viral infection across the blood brain barrier, using infected leukocytes, or by retrograde transport along cranial or peripheral nerves. ${ }^{4}$ The inflammatory cytokine storm resulting from the host innate immune response to

Copyright $(C 2021$ The Author(s). This is an open-access article distributed under the terms of the Creative Commons Attribution License (http:// creativecommons.org/licenses/by/4.0), which permits unrestricted use, distribution, and reproduction in any medium, provided the original work is properly cited. 
Table 1. Summary of Neuropsychiatric Manifestations and Sequelae of COVID-19

\begin{tabular}{ll}
\hline Category of Neuropsychiatric Disorder & Clinical Features/Syndromes \\
\hline Encephalopathies & Headache, vertigo, encephalitis, coma, seizures, ataxia, acute disseminated encephalomyelitis \\
Cranial nerve lesions & Anosmia, ageusia, internuclear ophthalmoplegia, optic neuritis, sixth nerve palsy, \\
\hline Peripheral neuropathies & $\begin{array}{l}\text { Guillain-Barré syndrome, Miller-Fisher syndrome } \\
\text { Ischaemic stroke, intracerebral haemorrhage, subarachnoid haemorrhage, cerebral dural venous sinus thrombosis, CNS } \\
\text { Cerebrovascular disease }\end{array}$ \\
Mental health disorders & Psychosis, affective disorders, delirium, dementia-like syndrome \\
Other & Generalised myoclonus, myopathy, myelitis, myelopathy \\
\hline
\end{tabular}

SARS-CoV-2 infection may damage neurologic tissue, as may cytotoxic $\mathrm{T}$ lymphocytes and antibody-mediated responses secondary to molecular mimicry between pathogen and host epitopes. ${ }^{4}$ A further mechanism may involve blood vessel damage from direct infection, immune-mediated vasculitis, endothelial activation, and thrombotic microangopathy. ${ }^{4}$

The burden of mental health problems precipitated by the social isolation associated with periods of societal lockdown has become one of the defining characteristics of this and other recent viral pandemics. Additionally, some patients with COVID-19 may present atypically with acute mental health disturbances. Altered mental status with an acute confusional state in patients infected with SARS-CoV-2 has led to use of the descriptive term 'COVID-19 delirium'. Recently published data from the UK have revealed the burden of psychiatric diagnoses among COVID-19 patients, including new-onset psychosis, a dementia-like neurocognitive syndrome, and affective disorders. ${ }^{7}$ These mental health disturbances may be temporally separated from the initial somatic symptoms of the disease and therefore may not be causatively linked with the infection.

The major neuropsychiatric features of COVID-19 are summarised in Table 1. While primarily an infection of the respiratory tract, COVID-19 has challenged clinicians with its atypical clinical presentations. Further research over time will help to elucidate their neuropathogenesis and enhance our understanding of this virus. As public fatigue with pandemic restrictions inevitably sets in, especially in younger cohorts, greater public awareness of the potential for long-lasting neurological sequelae is needed, to remind people of the continuing need to avoid contracting and/or spreading this virus. The so-called 'Spanish flu' left its indelible mark on the world. What historical lessons emerge from this pandemic remain to be seen.

\section{Authors' Contributions}

GTF conceived the idea for the manuscript. WHC, NN and GTF researched the material. GTF prepared the first draft of the manuscript which was edited for significant intellectual content by NN and WHC. All authors approved the final version of the manuscript.

\section{Conflict of Interest Disclosures}

Gerard Thomas Flaherty serves as a editorial board of International Journal of Travel Medicine and Global Health. Other authors declare that they have no conflicts of interest.

\section{Ethical Approval}

Not applicable.

\section{Funding/Support}

None.

\section{References}

1. Zubair AS, McAlpine LS, Gardin T, Farhadian S, Kuruvilla DE, Spudich S. Neuropathogenesis and neurologic manifestations of the coronaviruses in the age of coronavirus disease 2019: a review. JAMA Neurol. 2020;77(8):1018-1027. doi:10.1001/ jamaneurol.2020.2065.

2. Agarwal A, Pinho M, Raj K, et al. Neurological emergencies associated with COVID-19: stroke and beyond. Emerg Radiol. 2020:1-8. doi:10.1007/s10140-020-01837-7.

3. Fiani B, Covarrubias C, Desai A, Sekhon M, Jarrah R. A contemporary review of neurological sequelae of COVID-19. Front Neurol. 2020;11:640. doi:10.3389/fneur.2020.00640.

4. Ellul MA, Benjamin L, Singh B, et al. Neurological associations of COVID-19. Lancet Neurol. 2020;19(9):767-783. doi:10.1016/ s1474-4422(20)30221-0.

5. Izquierdo-Domínguez A, Rojas-Lechuga MJ, Chiesa-Estomba C, et al. Smell and taste dysfunction in COVID-19 is associated with younger age in ambulatory settings: a multicenter cross-sectional study. J Investig Allergol Clin Immunol. 2020;30(5):346-357. doi:10.18176/jiaci.0595.

6. Moein ST, Hashemian SM, Mansourafshar B, Khorram-Tousi A, Tabarsi P, Doty RL. Smell dysfunction: a biomarker for COVID-19. Int Forum Allergy Rhinol. 2020;10(8):944-950. doi:10.1002/ alr.22587.

7. Varatharaj A, Thomas N, Ellul MA, et al. Neurological and neuropsychiatric complications of COVID-19 in 153 patients: a UK-wide surveillance study. Lancet Psychiatry. 2020;7(10):875882. doi:10.1016/s2215-0366(20)30287-x. 